\title{
Enhanced detection and isolation of angle of attack sensor faults
}

\author{
Daniel Ossmann * \\ Department of Aerospace Engineering \& Mechanics \\ University of Minnesota, Minneapolis, MN, 55455, USA \\ Hans-Dieter Joos ${ }^{\dagger}$ \\ Institute of System Dynamics \& Control \\ German Aerospace Center (DLR), 82234 Wessling, Germany
}

\begin{abstract}
An enhanced detection and isolation method to monitor the state-of-practice triplex redundant angle of attack measurements on modern civil transport aircraft is presented. The developed fault detection and diagnosis architecture relies on advanced model and signal based techniques monitoring each of the three sensors individually. This allows the correct isolation of erroneous sensors also in case of multiple sensor faults. The gathered isolation information is used in an advanced sensor fusion scheme, allowing the propagation of an adequate angle of attack value to the flight control computer in case of failure. The fault detection and diagnosis system is validated using a high fidelity benchmark model of a large commercial transport aircraft using different wind excitations together with challenging pilot and auto-pilot scenarios.
\end{abstract}

\section{Introduction}

The angle of attack value is an inevitable measurement in modern fly-by-wire aircraft to obtain full flight envelope protections in the whole operational domain of the aircraft. Triplex sensor redundancy is used on modern aircraft to provide a fault tolerant signal fusion scheme. This special kind of fusion scheme is called signal consolidation and generates one single consolidated value out of the three sensor signals and propagates the value to the flight control system (FCS), which generated commands to the control surfaces. In parallel to the signal consolidation scheme each of the sensors signals is compared to the consolidated value and discarded if its deviation from the consolidated value is becoming too large. This state-of-practice signal consolidation with its monitoring scheme is compliant with the stringent regulations demand by the aircraft industry during the certification process. However, this signal based monitoring based on hardware redundancy has one significant drawback: in case of more than one simultaneous sensor fault, which in reality is rather improbable but not impossible, the system may discard the correct signal and process the faulty measurements to the FCS. Subsequently, erroneous signals could be sent to the control surfaces. This could lead to an abnormal and non-optimized behavior of the aircraft, causing an increased drag which goes hand in hand with a higher fuel consumption. Thus, a fast detection and isolation of the sensor faults could guarantee an optimal operation of the aircraft in case of faults.

The improvement of the state-of-practice fault detection scheme using different approaches is a widely studied topic in literature. In Ref. 1 a so-called soft signal consolidation scheme based on Fuzzy logics together with a Kalman filter for oscillatory failure detection in air-data and inertial sensor measurements is presented. In Ref. 2 the model based monitoring of inertial yaw rate sensors using sliding mode observers, including the validation on a high fidelity benchmark with different pilot inputs, is shown. Basic kinematic equations of the aircraft dynamics are applied in Ref. 3 to monitor inertial and air data measurements.

*Postdoctoral Associate: DOssmann@umn.edu

†Research Scientist: Dieter.Joos@dlr.de 
A virtual sensing based on analytical redundancy is applied to monitor the angle of attack and velocity measurements is shown in Ref. 4. The approach is validated using simulation and real flight test data.

All these articles present adequate approaches using model based techniques and even show the validation using simulation and real flight test data. However, these model based approaches generally use filter showing the same order as the underlying dynamics, which is critical when it comes to the implementation of the filters on flight control computers, due to the limited computational power. Further, the higher the order of the fault detection filter is, the more it relies on the mathematical approximation of the aircraft, making it more sensitive to model uncertainties. Thus, in this paper we will use a model based technique to generate a residual filter of minimal order, relying on the approaches presented in Ref. 5.

Strong arguments why model based fault detection techniques should be used are rarely found in the common fault detection literature. An argument that is often used to justify the use of model based fault detection is, that it outperforms signal based fault detection methods in detection performance and robustness. By signal based methods we refer to fault detection methods which only process the available signals without making use of the underlying model dynamics or any kinematic equations. However, the mentioned statement is only partly true: due to unmodeled dynamics, model uncertainties and stochastic noise inputs, the decision making process in model based fault detection if a fault is present or not, is highly dependent on the selected thresholds. The larger this threshold has to be selected to avoid false alarms, the more the detection performance in terms of minimum detectable fault amplitude and detection time is degraded. In contrast to that, signal based methods as limit checking are mathematically simple and independent of any analytical models. However, faults that may lie in the operational range of the system may remain undetected. In these cases it is not possible to distinguish between normal and faulty behavior by using signal based methods. These are the situations where model based approaches show their strength. Thus, when facing the decision whether to use model or signal based approaches the question about the fault to be detected has to be raised: if the fault lies in the normal operation range (amplitude, slope, variance etc.) of the measured system parameter to be monitored, then model based fault detection approaches can make a great advantage, as such faults cannot be detected by signal based methods. This may be true for very slow runaways, oscillatory failures with frequencies below system bandwidth and small biases. On the other side, if faults need to be detected which are beyond the operational range of the system (e.g. runaways with very high rates, high noise ratios) then a simple look at the characteristics of the sensor signal may be sufficient to come to the conclusion that the sensor is malfunctioning.

In this paper the goal is to develop a reliable fault detection and diagnosis (FDD) system to monitor the triplex redundant angle of attack (AoA) measurements on a civil transport aircraft. This problem has been defined as part of the EU-FP7-RECONFIGURE project. ${ }^{6}$ Various different fault types with different parameter settings have to be detected, some of them lying inside, some of them lie outside the normal operational behavior of the AoA. A FDD architecture is developed which makes use of model and signal based techniques to monitor the sensors. The proposed FDD architecture has an explicit advantage over the state-of-practice signal consolidation and voting based monitoring: it allows the individual monitoring of each sensor, implicitly solving the fault isolation problem even in case of multiple sensor faults. In this way it is possible to select the remaining healthy sensors and propagate a fault free AoA value to the FCS.

\section{Problem definition}

Current industrial state-of-practice uses a triplex voting scheme in fault free situations to generate the socalled consolidated value, ${ }^{1,6}$ which is propagated to the FCS. One implementation of this signal consolidation is to take a weighted median value of the three sensor sources defined by

$$
\alpha_{c}=\frac{1}{2} \operatorname{median}(\bar{\alpha})+\frac{1}{4}(\min (\bar{\alpha})+\max (\bar{\alpha}))
$$

where $\alpha_{c}$ is the consolidated AoA value and $\bar{\alpha}$ a vector containing the three AoA measurement signals $\alpha_{1}$, $\alpha_{2}$ and $\alpha_{3}$. In parallel a defined value $\pm \tau$ is added to the consolidated value to define the validity range of the sensor signals. If one of the signals crosses the lower or upper threshold the sensor is assumed to be faulty and the signal is discarded. Then the consolidated value is computed as the mean of the remaining two sensor signals

$$
\alpha_{c}=\frac{1}{2} \sum_{k=1}^{3} \alpha_{k} i_{k}
$$


where $i_{k}$ is a boolean variable, indicating if the $i^{\text {th }}$ sensor lies within the validity region $\left(i_{k}=1\right)$ or not $\left(i_{k}=0\right)$. The monitoring of the two remaining sensors is based on the difference between the two sensor signals. If their difference exceeds $2 \tau$, a failure in one of the sensors is assumed and the consolidated value is fixed to the last valid sensor signal, as fault isolation, i.e., the exact localization which sensor is faulty, is not possible using this strategy. The described working principal is depicted in Fig. 1.

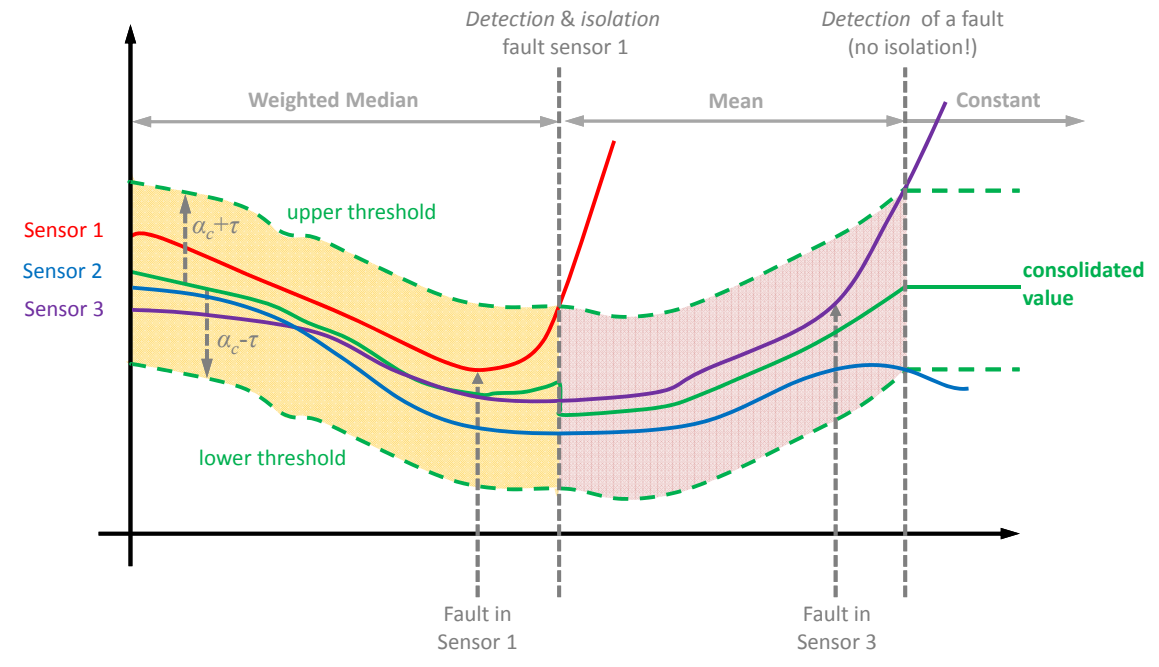

Figure 1. State-of-practice sensor consolidation and monitoring based on triplex redundancy

This scheme is able to provide a correct flight AoA value in fault free as well as single sensor fault scenarios. However, the scheme may be insufficient in multiple sensor fault scenarios. As mentioned above, if a second sensor fault appears after the first fault has been detected, the last valid value is propagated to the flight control system, which may cause a degradation of the protection performance. In the case of two simultaneous sensor faults an additional problem appears: as the decision variables $i_{k}$ implicitly depend on all three sensor signals via the consolidated value and its computed validity range, the correct sensor signal could be sorted out while the erroneous sensor signals may be propagated. This becomes even more severe when considering three simultaneous faults of the same kind: due to the inherent dependency of the validity region on the three measurement signals, the fusion scheme considers all three sensors as fault free as none of the sensor signals leaves the validity region.

Thus, the purpose of this work is to enhance the functionality of the state-of-practice fault detection scheme by introducing an additional individual monitoring of each sensor without using the sensor information of the other two sensors. Thus, in this approach each decision variable $i_{k}$ only depends on the k-th AoA sensor signal and is decoupled from the other two AoA sensor signals. An update of the signal consolidation scheme based on (2) for the case of one or two faults when using this advanced fault detection and isolation (FDI) scheme can be stated as:

$$
\alpha_{c}=\sum_{j=1}^{3} \alpha_{k} i_{k} / \sum_{j=1}^{3} i_{k}
$$

While the scheme is the same as before for the fault free cases, the mean based signal consolidation is updated for the multiple fault case: it simply multiplies each sensor signal with its decision variable and divides the sum by the number of correct values. This is now possible as three independent decision variables are generated. Note, in case of three sensor faults a correct detection and isolation of three sensor faults is provided, but no correct AoA value can be propagated to the FCS any more. However, if desired, the last correct value could be propagated as in the original approach.

The main question to be answered within this paper is how to robustly provide the decision variables $i_{k}$ based on model based and signal based methods. The AoA of the aircraft is a so-called air data measurement, i.e. it is measured by sensors which are placed outside the aircraft and are directly influenced by external influences as for example wind and temperature. Thus, a faulty behavior of these sensors may be the result of external influences, which may also induce faults on other air data measurements as the velocity. Due 
to this fact, as additional constraint the FDD system to monitor the three AoA sensors should not use any other measurements from the air data measurement system.

In many contributions in the existing literature the fault detection system is developed for one dedicated type of sensor fault as oscillatory faults, biases or drifts to just name a few of them. In the problem definition of the EU-RECONFIGURE project the aim is to detect and isolate a variety of sensor faults, which can appear on each of the three sensors, either as single or multiple fault event. The only constraint is, that the appearing fault type is the same on all three sensors. This is a realistic assumption when thinking for example about frozen sensor signals due to icing. However, the detection performance of the proposed FDD system is not sensitive to the combination of the appearing faults types, as each sensor is monitored individually. It just limits the fault scenarios to be tested in the validation process.

The relevant sensor fault types defined in the RECONFIGURE project to be detected are listed in Table 1. Note, that the sensor bias and the sensor drift have been separated in small and large biases and slow and fast drifts, respectively. As mentioned in the introduction, model detection is necessary if a behavior in the sensor signal is induced by the fault, which can appear in the signal also during normal operational conditions. This is true for small sensor biases and slow sensor drifts. Large biases, oscillatory failures, fast drifting, extensive sensor noise, non-return-to-zero as well as frozen sensor signals induce signal characteristics which directly indicate a faulty sensor. In these cases the provided measured value may be unrealistically large or the increase in one single time step exceeds the physical behavior of the plant. Thus, these faults will be detected by dedicated signal based methods.

Table 1. Different sensor faults to be detected

\begin{tabular}{ll} 
Failure type & Parameter \\
\hline \hline small bias & between 0.5 and $5 \mathrm{deg}$ \\
slow sensor drift & with slope magnitude between 0.2 and $10 \mathrm{deg} / \mathrm{s}$ \\
\hline large bias & between 5 and $180 \mathrm{deg}$ \\
freezing & at current value \\
oscillatory failure & with an amplitude of $0.5-25 \mathrm{deg}$ at $0.5-1 \mathrm{~Hz}$ \\
fast sensor drift & with slope magnitude between 10 and $25 \mathrm{deg} / \mathrm{s}$ \\
excessive sensor noise & with a standard deviation between 0.4 and $4 \mathrm{deg}$ \\
non-return-to-zero & random sequence of step signals with a minimum amplitude of $2 \mathrm{deg}$
\end{tabular}

The actual cause of these sensor faults are not the main topic of this paper. They may result from malfunctions of the sensors itself (as short-circuits), of the sensor's heating, of the signal conversion from analog to digital or simply from aging or external influences as temperature or dust, just to name a few examples.

\section{FDD system architecture}

Based on the considerations of the last section we propose an advanced fault detection and diagnosis architecture to monitor the three AoA sensors individually as proposed in Refs. 1, 7, i.e. not using the redundant measurement signals for the monitoring of a sensor. Thus, the proposed FDD architecture consists of three individual FDD systems which use as inputs, beside the angle of attack, the measurement signals provided by the internal measurement unit (IMU) as well as the commanded control inputs to the actuators from the flight control system (FCS). Such an FDD architecture is depicted in Fig. 2. Each of the three FDD systems provides a decision variable if the sensor is healthy $\left(i_{k}=1\right)$ or not $\left(i_{k}=0\right)$. As each system does not use the redundant measurement signals of the AoA, the fault isolation problem is solved implicitly. The three FDD systems consist of a model based and signal based part to monitor the corresponding AoA sensors. The theoretical background of their design and functionality is described in the following sections.

\section{A. Model based component}

The approach for the design of the model based FDI algorithm relies on advanced nullspace computations of the underlying system. The idea of the nullspace computation based FDI goes back to Ref. 8 and has been 


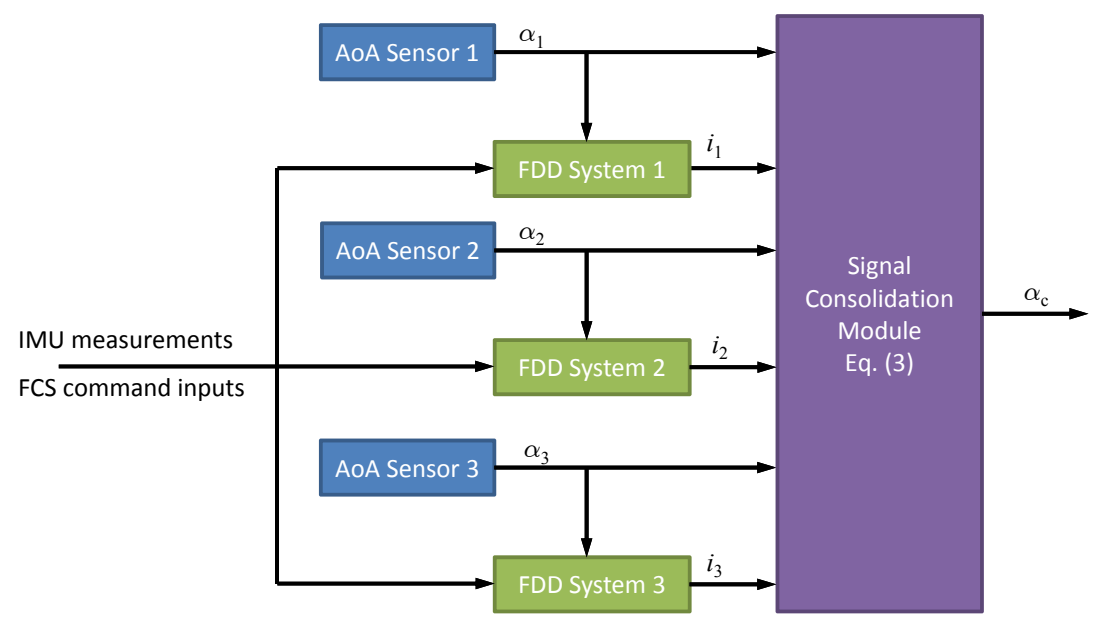

Figure 2. Fault detection and diagnosis architecture

improved and updated in various directions. The tools used in this paper are based on the work in Ref. 9 which make use of descriptor system methodology for a better numerical stability of the nullspace computation. The main advantages of the nullspace based residual filter design lies in the possibility to design filters of minimal order, providing a minimal dependency of the underlying dynamical model and thus, decreasing the influence of model uncertainties. Another advantage is the possibility to directly decouple disturbances from the residuals.

The general input-output form of an arbitrary linear system can be stated as

$$
\mathbf{y}(s)=G_{u}(s) \mathbf{u}(s)+G_{d}(s) \mathbf{d}(s)+G_{f}(s) \mathbf{f}(s),
$$

where $\mathbf{y}(s), \mathbf{u}(s), \mathbf{d}(s)$ and $\mathbf{f}(s)$ are the Laplace-transformed quantities of the system output $y(t)$, the control input $u(t)$, the disturbance input $d(t)$ and the fault input $f(t)$, respectively, and $G_{u}(s), G_{d}(s)$ and $G_{f}(s)$ are the corresponding transfer matrices. To solve the fault detection and isolation problem for the system (4) the residual filter

$$
\mathbf{r}(s)=Q(s)\left[\begin{array}{l}
\mathbf{y}(s) \\
\mathbf{u}(s)
\end{array}\right]
$$

shall be generated, which uses the available command input vector $u$ and the measured output vector $y$ of the system to generate a residual vector. In (5) $\mathbf{r}(s)$ is the Laplace-transformed quantity of the residual vector $r(t)$. Note, for the case of fault detection usually a scalar output may be sufficient, while for fault isolation so-called structured residuals are used. ${ }^{10}$ As the strategy in this paper is to monitor the three AoA sensors individually without using the redundant AoA sensor signals, three fault detection filters, each with a scalar output, are designed. Each of them solves the fault detection problem (FDP) for one of the sensor faults, while fault isolation is achieved implicitly by only using one AoA measurement for each filter. In what follows we will described how to solve the FDP for one filter, as the procedure is equivalent for all three filter designs.

The idea of the nullspace methodology becomes clear when inserting Eq. (4) into the residual filter (5):

$$
\mathbf{r}(s)=Q(s)\left[\begin{array}{cc}
G_{u}(s) & G_{d}(s) \\
I & 0
\end{array}\right]\left[\begin{array}{l}
\mathbf{u}(s) \\
\mathbf{d}(s)
\end{array}\right]+Q(s)\left[\begin{array}{c}
G_{f}(s) \\
0
\end{array}\right] \mathbf{f}(s)
$$

The residual shall be zero in any fault free situation while it has to be non-zero if a fault occurs. The residual will only be zero in case of no fault $(f=0)$ if it is decoupled from disturbances and inputs. Thus, the residual filter $Q(s)$ must guarantee

$$
Q(s)\left[\begin{array}{cc}
G_{u}(s) & G_{d}(s) \\
I & 0
\end{array}\right]:=Q(s) G_{e}(s)=0,
$$


implying that $Q(s)$ belongs to the left nullspace of $G_{e}(s)$. Beside this decoupling characteristics of the command and disturbance inputs, $Q(s)$ must provide good detection characteristics, i. e. $r$ must be coupled with the fault $(f \neq 0 \rightarrow r \neq 0)$. The design process is as follows: find all possible basis vectors of the nullspace of $G_{e}(s)$ and check in the second step if the fault is coupled or not. Those basis vectors which couple the fault to the residual and have minimal order are of special interest. Different methods exist to find a minimal bases of a system. For example the approach in Ref. 8 is based on finding minimal polynomial bases, while in Ref. 11 algorithms to directly compute minimal rational bases based on orthogonal reduction techniques are used. We will assume in this paper that a minimal basis has been found. Additionally, for a physically realizable filter, $Q(s)$ must be proper and stable, having only poles with negative real parts. The poles of the filter are included in the design freedom. The only design limitation is given by the (minimal) order of the filter which is constrained by the order of the nullspace basis.

The proposed model based FDI approach to design the FDI filter includes two steps: The preliminary design step is based on the presented nullspace method. For a set of linearized models the nullspace method is applied to gather information of the required residual filter structure. This structure is then used in a second step, namely a multi-objective turning using nonlinear simulation data. The advantage of the preliminary step is that filters of minimal order with a reduced set of input parameters can be generated. Implicitly, the complexity of the optimization problem for the second step is reduced. By this approach the goal of generating a LTI filter of low complexity providing good detection performance in terms of fault detectability and robustness against model uncertainties in the whole operational range of the aircraft can be achieved.

\section{Preliminary selection of basis vectors}

In this section the approach how to apply the nullspace computation to gather information about the filter structure is presented. We are assuming, that a basis $N_{l}$ of minimal order of the left nullspace of $G_{e}(s)$ has been found. $N_{l}$ has the dimension $q \times\left(m_{u}+m_{y}\right)$, where $m_{u}$ is the number of control inputs, $m_{y}$ the number of measurement signals and $q$ defines the number of basis vectors of the nullspace $N_{l}$. According to Ref. 10 the number of rows is given by $q=m_{u}+m_{y}-\operatorname{rank}\left(G_{e}\right)$.

While in literature the determination of the minimal nullspace basis is widely discussed, there is a lack of advise who to select the basis vectors with respect to their robustness against model uncertainties. Assuming two basis vectors having the same (minimal) row degree and showing the same transfer gain of the fault to the residual, the question remains, which basis vector should be selected as residual filter. Ref. 12 proposes to mix the basis vectors to achieve the best possible fault detectability when the FDP needs to be solved for the multiple faults case, i.e., when all possible faults should be coupled on single detector output. However, if only one single fault shall be detected the mixing of all possible basis vectors seems questionable, as the use of more basis vectors can unnecessarily introduce a higher dependency on the underlying model and thus, increase the influence of the uncertainties as unmodeled dynamics or approximation errors on the residual. These uncertainties lead to a non-perfect decoupling of the inputs and disturbances from the residual causing undesired residual excitations in fault free scenarios.

Let $\tilde{N}_{l}$ be the reduced nullspace matrix including the row vectors of $N_{l}\left(G_{e}(s)\right)$ which have minimal order and couple the fault $f$ to the residual $r$. We select a diagonal $p \times p$ matrix $\tilde{H}(s)$ to scale $\tilde{N}_{l}\left(G_{e}(s)\right)$ so that its rows are all proper and stable, show the same dynamics and a fault-to-residual dc-gain of $1 . p$ is the number of rows of $\tilde{N}_{l}$. As the poles of the filter $Q(s)$ can be freely chosen and the rows of $\tilde{N}_{l}$ have the same row degree, generating stable and proper rows with the same dynamics can always be achieved. The scaling of the fault-to-residual transfer behavior remains to be a constant scaling. In this way, each row of $\tilde{H}(s) \tilde{N}_{l}$ is a possible detector of minimal order solving the FDP. As we want to reduce the influence of uncertainties on the residual we choose our detector as based on the basis vector with the lowest induced $L_{2}$ norm from the filter inputs to its output. By taking the $L_{2}$ norm, the inputs of the filter are assumed to be physically uncorrelated, thus giving a measure how input uncertainties influence the filter output in the worst case. Minimizing the induced $L_{2}$ norm is equivalent to minimizing the $H_{\infty}$ of a transfer function, namely

$$
\min _{i} \frac{\left\|r_{i}\right\|_{2}}{\left\|\left[\begin{array}{ll}
y^{T} & u^{T}
\end{array}\right]^{T}\right\|_{2}}=\min _{i}\left\|\tilde{h}_{i}(s) \tilde{N}_{l}(s)\right\|_{\infty},
$$

where $\tilde{h}_{i}(s)$ are the rows of $\tilde{H}(s)$. Thus, by solving (8) and finding the parametrization vector $h_{i}^{*}(s)$ generating the detector with the lowest $H_{\infty}$ norm, the corresponding detector can be directly computed by $Q(s)=$ $\tilde{h}_{i}^{*}(s) \tilde{N}_{l}(s)$. By choosing this detector, the influence of uncertainties on the residual are decreased. 
Note that the selection of the detector based on (8) does not necessarily give the detector with the lowest possible $H_{\infty}$ norm, but the detector with the lowest $H_{\infty}$ norm for the determined minimal basis $\tilde{N}_{l}$. As the minimal basis $N_{l}(s)$ is not unique, detectors with lower $H_{\infty}$ norms still showing minimal order characteristics could be available. A further reduction of the $H_{\infty}$ norm could be achieved by different linear combinations of the rows of $N_{l}(s)$, i.e. choosing a non-diagonal matrix $H(s)$ to create these detectors. However, for the application shown in this paper the norm could not be lowered and the (row) vector-wise evaluation of (8) of the determined nullspace basis $\tilde{N}(s)$ has been sufficient.

\section{Multi-objective tuning of the filter}

To improve the performance of the detector $Q(s)$, in a second step, the non-zero entries of the matrices $B_{Q}$ and $D_{Q}$ of the state space realization

$$
\begin{aligned}
\dot{x}_{Q} & =A_{Q} x_{Q}+B_{Q}\left[\begin{array}{ll}
y^{T} & u^{T}
\end{array}\right]^{T} \\
r & =C_{Q} x_{Q}+D_{Q}\left[\begin{array}{ll}
y^{T} & u^{T}
\end{array}\right]^{T}
\end{aligned}
$$

of the filter $Q(s)$ are retuned using simulation data obtained with a high fidelity nonlinear model. Let $\vartheta$ contain all the parameters to be tuned. Note, as the dynamics of the filter can be freely chosen $A_{Q}$ is fixed during the nullspace design process, while $C_{Q}$ can be kept constant as a corresponding scaling can always be achieved by manipulating $D_{Q}$. Further, the zero entries of the matrices $B_{Q}$ and $D_{Q}$ given by the preliminary design step are kept at zero, thus keeping the filter structure. To tune the parameters $\vartheta$ of the residual filter, nonlinear simulation data is generated on $N$ different trimming points in the operation domain. Different command and disturbance maneuvers are selected for the fault free operation to excite the residual due to model uncertainties. As optimization constraint the fault detectability, i.e. the transfer behavior from the fault-to-residual, needs to be maintained. Hence, the following optimization problem needs to be solved for an update of the filter parameters:

$$
\begin{array}{ccc}
\min _{\vartheta} & \max & \left|r^{(i)}(t, \vartheta)\right| \\
& t \in\left[0, t_{\text {end }}\right] \\
& i=1: N
\end{array}
$$

subject to

$$
\begin{aligned}
& \max _{t \in\left[0, \tilde{t}_{\text {end }}\right]}\left|\tilde{r}^{(i)}(t, \vartheta)-\mathcal{F}(f)(t)\right|<\epsilon \\
& i=1: N
\end{aligned}
$$

where $\epsilon$ is a desired accuracy of the fault estimation. The fault estimation error is the difference between the fault estimate $\tilde{r}^{(i)}$ and $\mathcal{F}(f)(t)$, which is the filtered version of the fault signal $f$ via the given fault-to-residual transfer-function $R_{f}(s)$. Note, the evaluation of one single flight point $i \in N$ in (10) and (11) during the optimization requires two simulations of the filter, namely one with and one without fault. Thus, in the two equations the residuals and the simulations times are named differently, namely $r$ and $\tilde{r}$ and $t_{\text {end }}$ and $\tilde{t}_{\text {end }}$, respectively.

\section{Implementation aspects}

As the output of the retuned residual filter $Q(s)$ will not be equal zero when implemented on the final hardware in fault free scenarios due to discussed reasons, the residual needs to be compared to a defined threshold $\tau_{m b}$ to decide if the sensor signal is corrupted by a fault or not. This threshold is selected by validating the residual in fault free scenarios featuring different disturbance and command inputs to the system. The maximum value of the residual in fault free situations serves as threshold. Note that the thresholds automatically limits the minimum fault amplitude to be detected by the residual filter. The decision variable $i_{m b, k}$, indicating if a fault on the $k^{\text {th }}$ sensor has been detected using the model based approach is generated comparing the absolute value of the residual to the threshold, $|r(t)|>\tau_{m b} \rightarrow i_{m b, k}=1$ and $i_{m b, k}=0$ otherwise, for $k=1,2,3$.

Another aspect to be considered for model based fault detection is, that the residual filters are designed for linear models, which are generated around certain trim point in the operational envelope. For systems with large operational domains this inevitably results in numerous different trim points the system can be 
operated on. On each of these trim points the underlying system dynamics can be different. To operate the residual filter correctly, the deviation from the current trim point has to be considered. This can be done by subtracting the current values of the aircraft from its lagged trim-values. A simplified version of this approach is used in this paper, namely a periodically executed, discrete reset to zero of the filter input parameters. This reset is executed only if the aircraft is flown in straight and level flight, which is validated by checking different (IMU) flight parameters, as the vertical load factor and pitch rate. Note that any measurements of the air-data system, as altitude or velocity, are explicitly excluded in this reset process to avoid a coupling of the FDD strategy from air data measurements.

Faults only to be detected by model based fault detection: small sensor bias, slow sensor runaway

\section{B. Signal based component}

Signal based fault detection approaches assume that the available signals contain information which directly indicates the presence of faults. The main advantage of such a signal processing based methods is their reduced complexity and the resulting low computational power requirements. The goal is to detect any fault before AoA protection functions are activated due to the fault. In what follows three dedicated methods are presented to detect certain malfunctioning of the sensors by signal processing. As the algorithms need to be implemented on a hardware, the methods are described in the discrete time domain.

\section{Discrete Fourier analysis}

A rigorous approach to identify oscillations in noise corrupted signals is the periodogram method, ${ }^{13}$ which is based on determining the power spectrum of a signal using the discrete Fourier transform (DFT). The DFT is easily computable using the fast Fourier transform (FFT) algorithm and allows a satisfactory accurate evaluation of the oscillation frequency together with strong statistical guarantee of the presence of the oscillatory signal. Still, the on-board implementation of FFT-based frequency analysis is questionable, due to the strict code certification requirements. To overcome these limitations, a recursive version of the DFT, as described in Ref. 14, can be used to detect oscillations in real-time, which has been applied for identifying oscillatory faults in aircraft actuators in. ${ }^{15}$ Let $T$ be the sampling period and let $n$ be the expected length of the time series $\alpha_{k}(i T)$, where $i=0,1,2 \ldots, n-1, k=1,2,3$ indicates the monitored AoA sensor. The DFT computes

$$
X(\omega)=\sum_{i=0}^{n-1} \alpha_{k}(i T) e^{-j \omega i T}
$$

for a given frequency $\omega$. The computation of $X(\omega)$ can be done recursively by defining the partial sum:

$$
Y_{m}(\omega)=\sum_{i=0}^{m} \alpha_{k}(i T) e^{-j \omega i T}
$$

and observing that

$$
Y_{m}(\omega)=Y_{m-1}(\omega)+\alpha_{k}(i T) e^{-j w m T},
$$

for $m=1, \ldots, n$. Evidently, $X(\omega)=Y_{n-1}(\omega)$, where (14) is initialized with $Y_{0}(\omega)=0$. An oscillation of frequency near $\omega$ is detected if $|Y(\omega)|>\tau_{\text {freq }}$, where $\tau_{\text {freq }}$ is a suitable threshold to be selected. In this way it is possible to specifically look for certain frequencies in the AoA sensor signals. An identified frequency which cannot be appear in fault free situations indicates a malfunctioning sensor. The decision variable $i_{o s c, k}$, indicating if a fault has been detected on the $k^{t h}$ sensor using the DFT is given by

$$
i_{o s c, k}= \begin{cases}1, & \text { if }|Y(\omega)|>\tau_{\text {freq }} \\ 0 & \text { otherwise }\end{cases}
$$

for $k=1,2,3$.

Faults to be detected efficiently with DFT: oscillatory failure 


\section{Limit and trend checking}

Limit and trend checking is a basic fault detection method which monitors the system parameters based on its measured values. As the topic of this paper are AoA sensor faults, possible faults in AoA sensors can be directly detected when checking the values of the sensors output. If the AoA sensor output exceeds certain operational limits $\alpha_{k}>\alpha_{\max }$ or $\alpha_{k}<\alpha_{\min }$ the sensor is about to deliver incorrect measurements. This basic signal based monitoring is only used as a backup method if other methods fail to detect the faults, as the faults are detected rather late, i.e., when the protection function have been activated already.

Trend checking via the signals rate of change is more important as high signal variations can indicate malfunctions of the sensors. A fault is detected if the sensor signal's rate of change exceeds its maximum fault free value: $\left|\Delta \alpha_{k}\right|>\Delta \alpha_{\max }$, where the rate of change can be approximated by

$$
\Delta \alpha_{k}=\frac{\alpha_{k}(i T)-\alpha_{k}((i-p) T)}{p T} .
$$

The parameter $p \geq 1$ is used to define the number of time steps over which the rate of change is determined. Larger values of $p$ might be necessary with the increasing of sensor noise to suppress its influence on the determined rate of change. These very simple rules allow a reliable detection of abnormal high rates of change as well as abnormal absolute values delivered by the sensor. The decision variable $i_{l c, k}$, indicating if a fault has been detected on the $k^{t h}$ sensor by checking its absolute and rate of change limits is given by

$$
i_{l c, k}= \begin{cases}1, & \text { if }\left(\left|\Delta \alpha_{k}\right|>\Delta \alpha_{\max }\right) \vee\left(\alpha_{k}>\alpha_{\max }\right) \vee\left(\alpha_{k}<\alpha_{\min }\right) \\ 0 & \text { otherwise }\end{cases}
$$

for $k=1,2,3$.

Faults to be detected efficiently with limit checking: large abrupt bias, fast sensor drift, oscillatory failure with high amplitudes, excessive sensor noise, non-return to zero

\section{Plausibility checks}

These kind of checks are an enhanced version of simply looking at the measurement signals as it also incorporates further signals of the system, as for example different inputs, without yet taking an analytical process model into account. A check which will be used in this paper is the fact, that there has to be a change in the AoA measurement signal - with some time delay taking the dynamics of the aircraft into account when the demand inputs of the actuators are changing. This can be used to detect the so called frozen sensor signals where the output does not change over time although the system's inputs do. Frozen sensor signals are usually hard to detect with model based approaches, as it requires sufficiently large excitations of the system, which can be specially difficult in aircraft operation when thinking of automated maneuvering by the autopilot, where the inputs usually a rather small and smooth.

One possibility among others to check an quantify an adequate input-output behavior is based on the so-called Narendra filter ${ }^{16}$ which approximates the 2-norm of a signal and has been successfully applied for aircraft actuator jamming detection and identification in Ref. 15. The filter is applied to the rate of change $\Delta \eta(t)$ of commanded elevator deflection as well as to the rates of change of three measurements to be monitored:

$$
\begin{aligned}
e_{\eta}(i T) & =\sum_{i=p}^{m} \Delta \eta^{2}(k T) \gamma_{\eta}^{i-p} \\
e_{\alpha_{k}}(i T) & =\sum_{i=p}^{m} \Delta \alpha_{k}^{2}(k T) \gamma_{\alpha}^{i-p}
\end{aligned}
$$

for $k=1,2,3$, where $m \geq p$ and $\Delta \eta$ can be determined according to (16) by

$$
\Delta \eta(i T)=\frac{\eta_{k}(i T)-\eta_{k}((i-p) T)}{p T} .
$$

An efficient recursive implementation of (18) is given by

$$
\begin{aligned}
e_{\eta}(i T) & =e_{\eta}((i-1) T) \gamma_{\eta}+\Delta \eta(i T) \\
e_{\alpha_{k}}(i T) & =e_{\alpha_{k}}((i-1) T) \gamma_{\alpha}+\Delta \alpha_{k}(i T)
\end{aligned}
$$


for $i \geq p$ and the initialization $e_{\eta}(i T)=0$ and $e_{\alpha_{k}}(i T)=0$ for $i<p$ respectively. Note that in case of more than one elevator on the aircraft the demanded deflections can be summed up to a single one. In (18) $\gamma_{\eta}>0$ and $\gamma_{\alpha}>0$ are the forgetting factors of the first order nonlinear filters. Note, the index for the different AoA sensors are missing at the parameters of the second line in Eq. (18) set, as for all three sensors the same parameter values are used. The decision variable $i_{p c, k}$, indicating if a fault has been detected on the $k^{t h}$ sensor by the plausibility check is given by

$$
i_{p c, k}= \begin{cases}1, & \text { if }\left\{\left(e_{\eta}>\tau_{\eta}\right) \wedge\left(e_{\alpha, k}<\tau_{\alpha}\right)\right\} \\ 0 & \text { otherwise }\end{cases}
$$

for $k=1,2,3$, where $\tau_{\eta}$ and $\tau_{\alpha}$ are thresholds to be selected. With an adequate setting of these thresholds together with the forgetting factors of the filters the noise influence in the measurements as well as the transfer dynamics of the underlying system can be taken into account. In words, the logic in (21) checks, if the energy of the rate of change at the input has a certain energy level. If so, there must exist an corresponding energy level on the sensors rate of change. An energy level of zero on the sensor rate of change together with a nonzero energy level of the input rate of change indicates a frozen sensor signal.

$\underline{\text { Faults to be detected efficiently with transfer logic: frozen sensor signal }}$

\section{Overall decision logic}

Finally, the decision variable $i_{k}$, indicating if the $k^{\text {th }}$ sensor is working correctly or not has is determined using an OR-gate:

$$
i_{k}= \begin{cases}1, & \text { if }\left\{\left(i_{m b, k}=0\right) \wedge\left(i_{o s c, k}=0\right) \wedge\left(i_{l c, k}=0\right) \wedge\left(i_{p c, k}=0\right)\right\} \\ 0 & \text { otherwise },\end{cases}
$$

In words, $i_{k}$ will be equal 1 if the sensor is working correctly and will be equal 0 if any fault type has been detected by one of the methods described above. With this boolean variable available, the signal consolidation scheme presented in Eq. (2) can be evaluated.

\section{Robust FDD system design}

In this section the application of the presented theoretical methodology on a modern, heavy-weight civil transport aircraft is presented. The designed FDD-architecture is validated using a non-linear high fidelity benchmark model, including nonlinear dynamics of the aircraft, the sensors and the actuators as well as the inner loop control laws, protection functions and different autopilot modes.

\section{A. Model based component}

A set of $N=214$ linear models generated on different points in the flight envelope and weight and balance domain is available for the design of the fault detection filter. Each of the model features seven IMU measurements, namely longitudinal and vertical load factor, pitch rate, ground speed, pitch angle, ground AoA, i.e. the AoA without wind influence, and altitude, as well as the corresponding AoA as the air data measurement to be monitored. The known inputs commanded by the FCS are the four elevator positions as well as the stabilizer position. In Tab. 2 the possible inputs to the residual filter $Q(s)$ are listed. Further, the linear models feature disturbance inputs to be decoupled, namely the wind in longitudinal and vertical direction as well as the commanded thrust, which is assumed to be not measurable.

Note that the $\delta$ is used to indicate that only the deviations from the trim values are used as inputs to the residual filter. Applying the first step of the procedure to design a model based fault detector as described in section A to the 214 models reveals the following structure of the residual filter:

$$
\begin{aligned}
A_{Q} & =-1 \\
B_{Q}^{(i)} & =\left[\begin{array}{lllllllllllll}
b_{1}^{(i)} & b_{2}^{(i)} & b_{3}^{(i)} & 0 & 0 & 0 & 0 & 1 & b_{9}^{(i)} & b_{9}^{(i)} & b_{11}^{(i)} & b_{11}^{(i)} & b_{13}^{(i)}
\end{array}\right] \\
C_{Q} & =1 \\
D_{Q}^{(i)} & =\left[\begin{array}{lllllllllllll}
0 & 0 & d_{3}^{(i)} & 0 & 0 & 0 & 0 & 0 & 0 & 0 & 0 & 0 & 0
\end{array}\right]
\end{aligned}
$$


Table 2. Available inputs of the $k^{t h}$ residual filter, for $k=1,2,3$

\begin{tabular}{rll} 
Index & Input signal & description \\
\hline 1 & $\delta n_{x}$ & Longitudinal load factor (body fixed x-direction) \\
2 & $\delta n_{z}$ & Vertical load factor (body fixed z-direction) \\
3 & $\delta q$ & Pitch rate \\
4 & $\delta V_{g}$ & Ground speed \\
5 & $\delta \alpha_{g}$ & Ground angle of attack \\
6 & $\delta \theta$ & Pitch angle \\
7 & $\delta h$ & Altitude \\
8 & $\delta \alpha_{k}$ & Angle of attack \\
\hline 9,10 & $\delta \eta_{l, i}, \delta \eta_{r, i}$ & Commanded left and right inner elevator deflection \\
11,12 & $\delta \eta_{l, o}, \delta \eta_{r, o}$ & Commanded left and right right outer elevator deflection \\
13 & $\delta i h$ & Commanded stabilizer position
\end{tabular}

The pole of the filter is selected at a value of -1 , leading to a fault to residual transfer behavior of $R_{f}(s)=$ $1 /(s+1)$ with a steady state gain of $R_{f}(0)=1$. Note that the entries for the two inner as well as for the two outer elevator deflections are identical, which is due to their symmetric positioning around $\mathrm{x}$ axis of the aircraft. The structure is the same for all 214 models, however showing different parameter values. As a constant filter needs to be designed, in a second step the best parameter values solving the optimization problem (10)-(11) are searched. As initialization of the parameters the mean value for each parameters over the 214 models are used and the set of free parameters for the optimization is defined as $\vartheta=\left\{b_{1}, b_{2}, b_{3}, b_{9}, b_{11}, b_{13}, d_{3}\right\}$. Note that by applying the nullspace based pre-computation of the filters and the basis vector selection, the set of free parameters to be optimized has been reduced to seven.

The data for the optimization is generated using the closed loop benchmark model including aircraft, actuator and sensor dynamics as well as the flight control system. This enables the simulation of the closed loop system with pilot and wind inputs. The pilot maneuver used for simulations is a stick doublet with $60 \%$ of the maximum stick deflection together with wind of $50 \%$ of the maximum wind to be considered in the benchmarks specifications, i.e. 20kts horizontal and 10kts vertical wind. The simulation time $t_{\text {end }}$ in (10) is equal to $120 \mathrm{~s}$. A second set of data is selected for the faulty case to be able to compute the corresponding residual signal $\tilde{r}(t, \vartheta)$ in (11). The AoA fault signal $f$ is a $5 \mathrm{deg}$ measurement bias starting at $t_{f}=10 \mathrm{~s}$. The simulation time $\tilde{t}_{\text {end }}$ is equal 40s. These two scenario are simulated on each of the 214 points in the flight envelope for which linear models are available. Solving the optimization problem finally enables to update the parameters of the residual filters state space matrices.

The implementation of the optimized residual filter requires the definition an adequate threshold $\tau_{m b}$. This threshold is usually determined using various numerical simulations of the nonlinear benchmark model. Alternatively, optimization based approaches can be used to determine the worst-case excitation of the residual in fault free cases. This worst-case excitation can serve as basis for the selection of the threshold. The advantage of this approach over the Monte-Carlo approach is, that it represents a target-orientated search for the adequate threshold, minimizing iteration and simulation costs of the FDD system synthesis procedure. ${ }^{17,18}$ The worst case search for different autopilot, smooth pilot and full wind inputs revealed that a threshold of $\tau_{m b}=3$ has to be used for the filter (23) to ensure no false alarms. Note that with the selection of the threshold the minimum detectable fault amplitude is automatically limited. With a value of $\tau_{m b}=3$ fault amplitudes below $3 \mathrm{deg}$ in the AoA measurements cannot be robustly detected.

\section{B. Signal based component}

The signal based methods described in section III.B are implemented for the monitoring of each of the three sensors. The specific values presented below are chosen by analyzing gathered simulation data of faulty and fault-free scenarios. They are selected to ensure a separation between these two scenarios.

For the oscillatory fault one frequency point showed to be sufficient together with a constant threshold 
$\tau_{\text {freq }}=200$. The window $n$ for the observation is selected at $n=1500$ in Eq.(12) which corresponds to 60 s at a sampling time of $T=0.04 \mathrm{~s}$. After $n$ time steps the DFT is reinitialized. For the trend checking the limits for the AoA are set to $\alpha_{\max }=25 \mathrm{deg}$ and $\alpha_{\min }=-10 \mathrm{deg}$, while the $\left|\Delta \alpha_{\max }\right|=10 \mathrm{deg} / \mathrm{s}$ with a the parameter $p=25$. For the plausibility checks by the Narendra filters in Eq. (20) the value of $p$ is kept at 25, while the forgetting factors are set to $\gamma_{\eta}=0.96$ and $\gamma_{\alpha}=0.96$ with the thresholds of $\tau_{\eta}=0.1$ and $\tau_{\alpha}=0.01$.

\section{Results}

To allow a realistic, industrial validation the designed FDD architecture the system is validated using a high fidelity benchmark model of the civil transport aircraft. This model is used in an extensive Monte Carlo campaign to verify the functionality of the approach. Additionally, a fault scenario based on a real in-flight incident is simulated.

\section{A. Industrial validation}

The implemented FDD architecture, depicted in Fig. 2, where each sensor is monitored individually using the described functions above, is validated using a high fidelity benchmark model. While designed on 214 specific design points with specific stick and wind inputs, the system is validated on randomly selected points in the flight envelope with a large variety different control and disturbance system inputs. Thus, the model is trimmed on random points of the flight envelope and weight and balance domain. To check the robustness against false alarms various maneuvers, including pilot inputs with different amplitudes, wind inputs with different wind velocities and gradients, turbulence inputs, wind shear as well as auto-pilot maneuvers are tested. All of the selected maneuvers did not show any false alarm in the fault free case.

The second step in the validation process is to test the detectability performance of the filter for different fault scenarios. As modern civil aircraft spends most of their operational time in steady state flight conditions as straight and level flights or climb/descent maneuvers with constant vertical speeds we assume that the fault appears during these flight conditions. Wind gusts, wind shear and turbulence may occur at any point during the operation of the aircraft. Note, that in case of full pilot excitation of the aircraft, the performance of the linear designed filter may be worse due to the fact, that the aircraft might be operated in a region, where the dynamics cannot be described by a linear model any more. In this case, an adaptive threshold is used to avoid false alarms, automatically degrading the fault detection performance characteristics of the model based fault detector. However, in case of smooth autopilot and/or low pilot inputs, the threshold can be kept low, providing a suitable detection performance. We consider the case when two AoA sensors fail at the same time. In case of only one fault the state-of-practice signal consolidation scheme is sufficient and can handle the loss of one sensor. However, when two or more sensors are lost, wrong measurement signals may be propagated to the FCS leading to a possible activation of the AoA protections if the propagated AoA reaches the protection limit.

In Tab. 4 the results of a statistical analysis of the fault detection times are shown. For each of the faults a Monte Carlo run with 200 simulations, again selecting random points of the flight envelope and weight and balance domain, is evaluated. The varying parameters are the aircraft mass, the center of gravity position, the initial altitude and the velocity as well as the time when the faults appears. Table 3 lists the minimum and maximum parameters for the Monte-Carlo simulations. All parameters are uniformity distributed over their parameter range. Note that the values for the minimum selectable speed (VLS) and maximum operating speed (VMO) are a function of the altitude and not given in more detail.

Table 3. Considered limits of the flight envelope

\begin{tabular}{lcc} 
Parameter & minimum value & maximum value \\
\hline Mass & $260 \mathrm{t}$ & $560 \mathrm{t}$ \\
Altitude & $5000 \mathrm{ft}$ & $35000 \mathrm{ft}$ \\
Center of gravity position & $28 \%$ & $44 \%$ \\
Calibrated Airspeed & $1.1 \mathrm{VLS}$ & $\mathrm{VMO}$ \\
Fault occurrence time & $5 \mathrm{~s}$ & $180 \mathrm{~s}$
\end{tabular}


Each simulation run is 250s long. The autopilot-maneuver with the demands depicted in Fig. 3 is defined to test the fault detection times: The aircraft starts in a straight and level flight on its trim altitude for $20 \mathrm{~s}$. Then, a level change of $2000 \mathrm{ft}$ with a vertical speed of $1200 \mathrm{ft} / \mathrm{min}$ and a decreased calibrated airspeed of $20 \mathrm{kts}$ is demanded. The new flight level is held for 20 s accelerating to the initial velocity. After, a level change of $-1000 \mathrm{ft}$ with a vertical speed of $-1000 \mathrm{ft} / \mathrm{min}$ with a calibrated airspeed 10kts higher than the initial one is demanded. For the remaining simulation time the altitude is kept constant. Wind turbulence with a standard deviation of 0.6 , corresponding to a maximum turbulence of $\pm 2 \mathrm{kts}$, is induced for the whole simulation. Additionally different wind gusts in longitudinal and vertical direction are induced during the simulation. The wind inputs in the longitudinal and vertical direction are depicted in the lower right diagram of the figure.
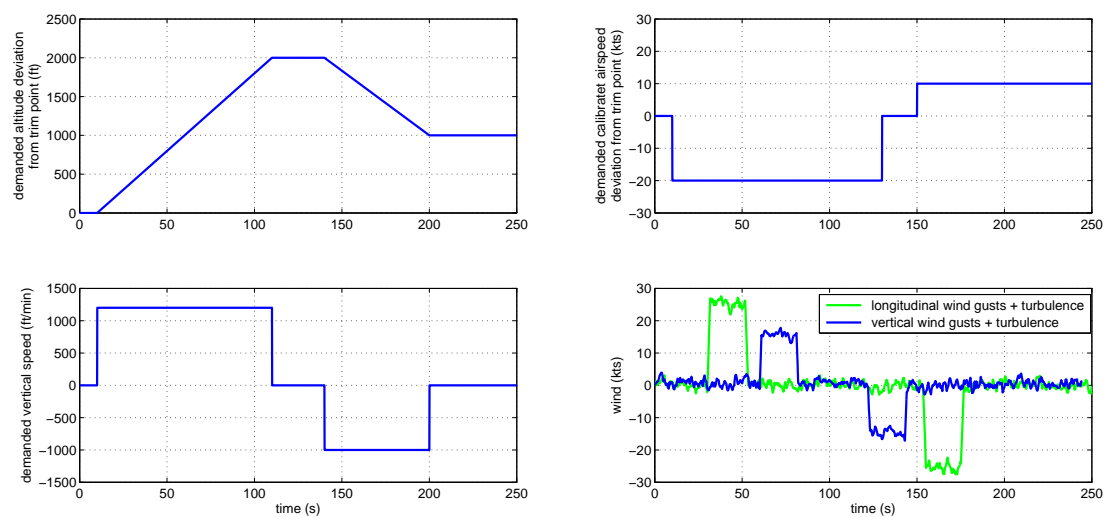

Figure 3. Flight maneuver to test fault detection performance

In Tab. 4 the Monte-Carlo analysis results for the detection times for different fault types are listed. Faults with high amplitudes and high rates of change are detected much faster than faults with low magnitude and low rates of change. This is evident, as the latter ones have to be distinguished from normal aircraft operation, while faults with high amplitudes/high rates of change can be detected fast due to the fact that they cannot appear in fault free operations of the sensors. A fault type difficult to be detected is the frozen sensor signal, which requires an adequate excitation of the aircraft dynamics for a successful detection. Small biases are hard to detect in certain situations, which is indicated by the maximum detection time of 14.6s. This large detection time is caused by not perfectly decoupled disturbances and command inputs, suppressing the fault influence in the residual for a certain time instance. Nevertheless, the mean detection time is satisfactory low, indicating that just at a very few number of flight points the delayed detection of the fault is given. Another challenging fault to be detected is the oscillatory failure with an amplitude of $2 \mathrm{deg}$. The low amplitude causes a delay in both, the signal based monitoring and the model based monitoring, resulting in a maximum value of 8.68s. However, as the magnitude of the fault is rather low, a detection time of over $8 \mathrm{~s}$ is acceptable. The detection times of the oscillatory failure notably decrease with an increase of the fault amplitude due to the fast exceeding of the threshold in the signal based monitoring scheme, enabling detection times below $0.1 \mathrm{~s}$

The results can be summed up as follows: The detection times for low fault amplitudes lying in the operational range of the AoA need some more time to be detected with the model based approach. This is due to the conservative selection of the threshold $\tau_{m b}$ ensuring no false alarm in the whole flight envelope. As the flight envelope is rather large, better performance could be reached in certain parts of the envelope when reducing the threshold in these regions. For simplicity one constant threshold has been selected for the validation process in this paper, as the primary goal was to provide a low complex FDD architecture minimizing the required computational power.

\section{B. Full flight simulation}

In this section the nonlinear benchmark will be used to simulate a full flight over approximately one hour without faults. The same flight profile is then simulated with two frozen sensor signals, causing the activation of the AoA protection. This scenario is inspired by the recently occurred incident of an commercial transport 
Table 4. Different sensor faults to be detected

\begin{tabular}{lccc} 
Failure type & Parameter setting & min $/$ max detection time & mean \\
\hline bias - small & $4 \mathrm{deg}$ & $0.67 \mathrm{~s} / 14.6 \mathrm{~s}$ & $1.71 \mathrm{~s}$ \\
bias - large & $8 \mathrm{deg}$ & $0.04 \mathrm{~s} / 0.08 \mathrm{~s}$ & $0.06 \mathrm{~s}$ \\
drift - slow & $1 \mathrm{deg} / \mathrm{s}$ & $2.11 \mathrm{~s} / 6.64 \mathrm{~s}$ & $4.05 \mathrm{~s}$ \\
drift - slow & $8 \mathrm{deg} / \mathrm{s}$ & $0.69 \mathrm{~s} / 1.33 \mathrm{~s}$ & $1.07 \mathrm{~s}$ \\
drift - fast & $25 \mathrm{deg} / \mathrm{s}$ & $0.06 \mathrm{~s} / 0.10 \mathrm{~s}$ & $0.07 \mathrm{~s}$ \\
excessive sensor noise & with a standard deviation of $0.6 \mathrm{deg}$ & $0.04 \mathrm{~s} / 0.31 \mathrm{~s}$ & $0.11 \mathrm{~s}$ \\
frozen sensor signal & at current value & $10.48 \mathrm{~s} / 10.92 \mathrm{~s}$ & $10.84 \mathrm{~s}$ \\
non-return-to-zero & with an amplitude of $2 \mathrm{deg}$ & $0.12 \mathrm{~s} / 0.24 \mathrm{~s}$ & $0.18 \mathrm{~s}$ \\
oscillatory failure - small & $2 \mathrm{deg}$ at $0.5 \mathrm{~Hz}$ & $3.08 \mathrm{~s} / 8.68 \mathrm{~s}$ & $7.46 \mathrm{~s}$ \\
oscillatory failure - large & $5 \mathrm{deg}$ at $0.5 \mathrm{~Hz}$ & $0.04 \mathrm{~s} / 0.08 \mathrm{~s}$ & $0.06 \mathrm{~s}$
\end{tabular}

aircraft where two AoA sensors signals got frozen due to external influence, which led to the activation of the AoA protection resulting in a short descent of the aircraft with an altitude loss of approximately $4000 \mathrm{ft} .{ }^{19}$ In the first diagram of Fig. 4 the flight profile is depicted. The aircraft is trimmed at around 6500ft with low speed. After an acceleration phase the aircraft climbs to its cruising altitude of 30000ft with 290kts in calibrated airspeed. After reaching the cruising altitude the aircraft is accented to the cruising speed of 320kts calibrated airspeed which is equivalent to a Mach number of 0.82 . After a short cruising phase the descent down to $5000 \mathrm{ft}$ with a vertical speed of $1100 \mathrm{ft} / \mathrm{min}$ and a calibrated airspeed of $270 \mathrm{kts}$ is started. After maintaining $5000 \mathrm{ft}$ for about two minutes the final descent is initiated. In the diagrams of the second row of the figure the corresponding consolidated AoA value and the Mach number are depicted. Note, due to the constant speed during the climb phase, the Mach number increases, leading to a decrease of the AoA activation limit (not shown in the diagram). In the last row of Fig. 4 the injected vertical wind gusts (left) and the longitudinal wind shear (right) both including additional turbulence are depicted.
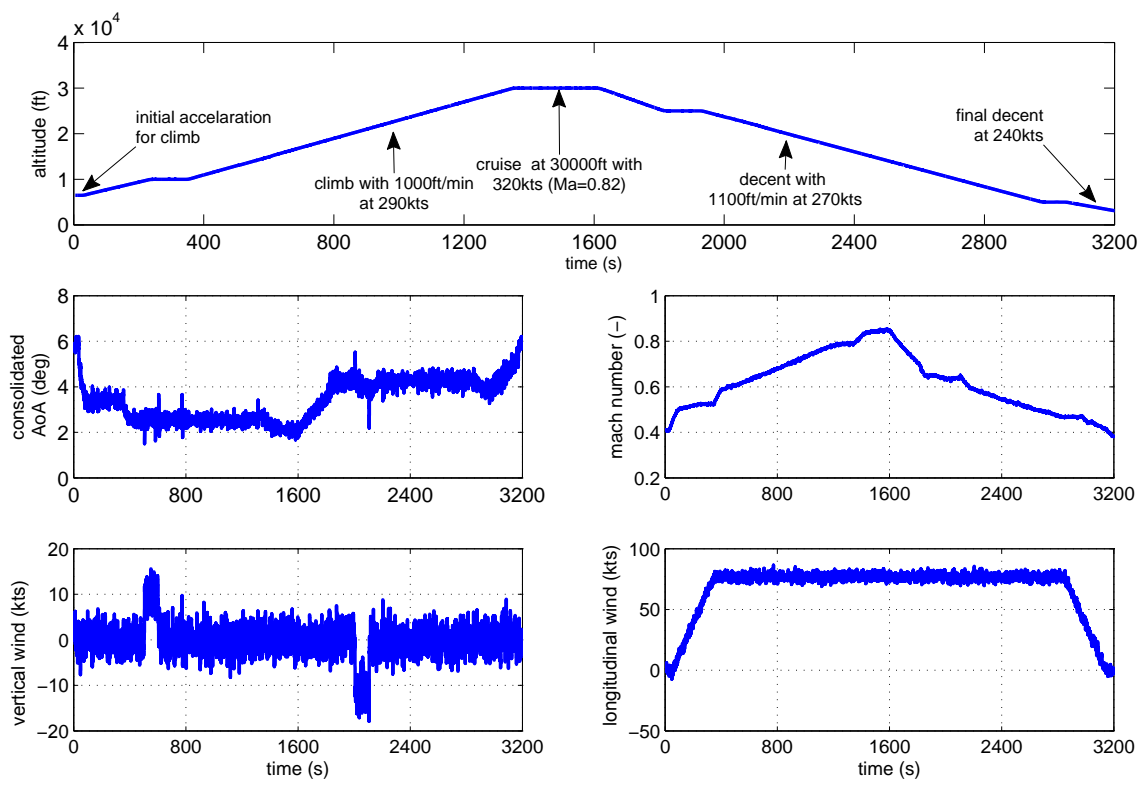

Figure 4. Simulated flight profile, consolidated AoA and Mach and defined wind inputs

In Fig. 5 the same flight is shown, however, this time the values of two of the three AoA sensors are 
frozen at $t_{f}=16 \mathrm{~s}$ when the climb is initialized. The scenario is simulated with the state-of-practice signal consolidation and monitoring system and with the proposed FDD system architecture. In the upper diagram of Fig. 5 the flight profile in case of the state-of-practice signal consolidation scheme (red) and the proposed scheme (blue) is depicted. Both profiles look equal to the profile of the fault free simulation in Fig. 4 until reaching 1300s simulation time. This is due to the fact that the AoA is not controlled directly by the FCS and thus, the faults have no influence as long as the consolidated AoA lies below the AoA protection limit. However, if the consolidated value exceeds the protection limit, the AoA protections are activated. As at constant calibrated airspeed the Mach number increases with altitude, the AoA protection limit, which is a function of the Mach number, decreases. In the lower right diagram of Fig. 5 the consolidated value of the state-of-practice signal consolidation is depicted in red. As two sensors are frozen, the consolidated value is equal to the outputs of the two frozen sensors, which is around 6deg. Thus the FCS believes that the actual AoA of the aircraft is 6deg for the whole simulation. With the decreasing of the AoA protection limit, at around 1400s the consolidated AoA value exceeds the protection limits, causing the activation of the AoA protections. This leads to a nose down command of the FCS to decrease the AoA, which is actually happening in reality but is not visible in the consolidated value as the sensors are still frozen. Note, the simulation has been aborted at this point as no realistic pilot model was available to stabilize the aircraft after the descent was initialized as it has been described in the description of the incident. ${ }^{19}$

In case of the proposed FDD system architecture the flight profile equals the fault free profile through the whole flight and the two frozen sensors do not lead to the activation of the AoA protection law. This is due to the successful detection and isolation of the two faults after about 10s after their occurrence, which can be seen in the lower left diagram of Fig. 4. Note, between the occurrence of the faults and their detection and isolation the consolidated value corresponds to the frozen value, as the median-based signal consolidation has been kept active until a fault was detected. As soon as two faults are detected the signal consolidation scheme is switched to the one presented in Eq. 3, where the two sensor signals are successfully suppressed from the AoA signal consolidation, avoiding the propagation of the faults to the FCS.
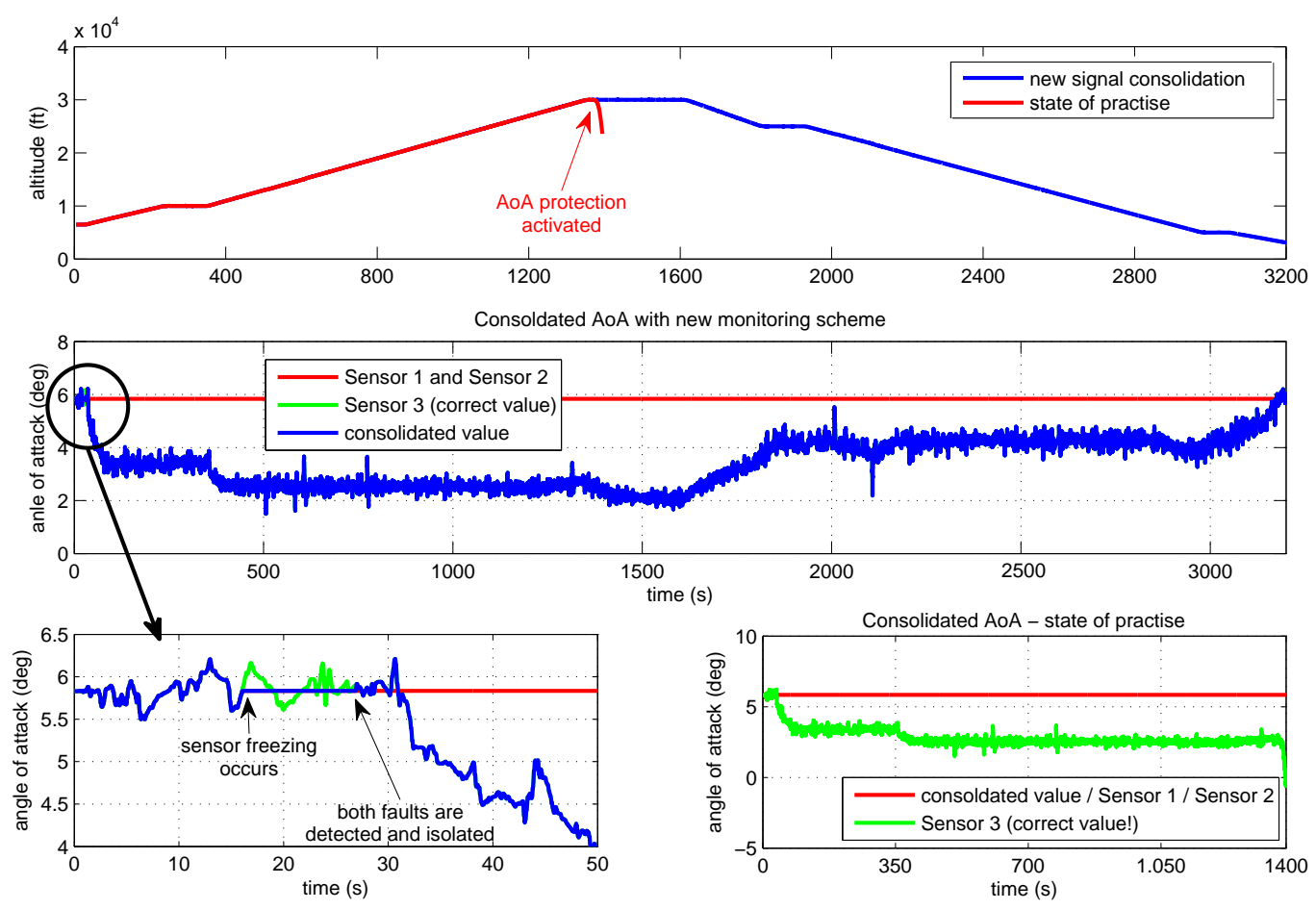

Figure 5. Simulated flight with two frozen AoA sensors, with and without reconfiguration 


\section{Conclusion}

An advanced fault detection and diagnosis (FDD) architecture to monitor the triplex redundant angle of attack air measurement unit of a commercial large transport aircraft has been presented. The FDD architecture incorporates modern signal and model based fault detection algorithms. Fault isolation is achieved by an individual monitoring of the three angle of attack (AoA) sensors using three FDD systems. Each system only uses one of the three redundant AoA sensor signals to achieve the desired fault isolation. The design of the linear residual filter is a two-step procedure in which the nullspace based fault detection synthesis method is used to determine the adequate structure of the fault detection filter, which is optimized using non-linear simulation data in the second step. While the procedure has been applied for the monitoring of AoA sensors it can be easily adapted for other sensor configurations. The designed FDD system has been validated on a high fidelity benchmark simulator of a large civil transport aircraft. The presented results indicate a potential enhancement of the current used monitoring schemes to provide adequate fault detection and isolation information also in case of multiple sensor malfunctions.

\section{Acknowledgment}

This work was performed in the framework of the European FP7 RECONFIGURE Project: Grant agreement AAT-2012-RTD-2314544.

\section{References}

\footnotetext{
${ }^{1}$ Berdjag, D., Cieslak, J., and Zolghadri, A., Fault Detection and Isolation of aircraft air data/inertial system, chap. Fault Detection and Isolation of aircraft air data/inertial system, Torus Press, 2013, pp. 317-332.

${ }^{2}$ Alwi, H. and Edwards, C., "Development and application of sliding mode LPV fault reconstruction schemes for the ADDSAFE," Control Engineering Practice, Vol. 31, 2014, pp. 148-170.

${ }^{3}$ v. Eykeren, L. and C, Q. P., "Sensor fault detection and isolation for aircaft control systems by kinematic realations," Control E, Vol. 31, 2014, pp. 200-210.

${ }^{4}$ Hardier, G., Seren, C., and Ezerzere, P., "Model-based techniques for virtual sensing of longitudinal flight parameters," International Journal of Applied Mathematics and Computer Sciences, Vol. 25, 2015, pp. 23-38.

${ }^{5}$ Varga, A., "On designing least order residual generators for fault detection and isolation," Proc. of 16th International Conference on Control Systems and Computer Science, Bucharest, Romania, 2007, pp. 323 - 330.

${ }^{6}$ Goupil, P., Boada-Bauxell, J., Marcos, A., Cortet, E., Kerr, M., and Costa, H., "AIRBUS efforts towards advanced real-time Fault Diagnosis and Fault Tolerant Control," Proc. of 19th IFAC World Congress, 2014.

${ }^{7}$ Varga, A., Ossmann, D., and Joos, H. D., "A fault diagnosis based reconfigurable longitudinal control system for managing loss of air data sensors for a civil aircraft," Proc. of IFAC World Congress, 2014.

${ }^{8}$ Nyberg, M., "Criterions for detectability and strong detectability of faults in linear systems," International Journal of Control, Vol. 75(7), 2002, pp. 490-501.

${ }^{9}$ Varga, A., "On computing nullspace bases - a fault detection perspective," Proc. of 17th IFAC World Congress, Seoul, South Korea, 2008.

${ }^{10}$ Varga, A., "On computing achievable fault signatures," Proc. of 7th IFAC Symposium on Fault Detection, Supervision and Safety for Technical Processes, Barcelona, Spain, 2009.

${ }^{11}$ Varga, A., "Numerically reliable methods for optimal design of fault detection filters," Proc. of Conference on Decision and Control, Seville, Spain, 2005.

${ }^{12}$ Varga, A., "A Fault Detection Toolbox for Matlab," Proc. of CASCD, Munich, 2006.

${ }^{13}$ Stoica, P. and Moses, R. L., Introduction to Spectral Analysis, Prentice Hall, 1997.

${ }^{14}$ Morelli, E. A., "Real-time parameter estimation in the frequency domain," AIAA Journal of Guidance, Control and Dynamics, Vol. 23, 2000, pp. 812-818.

${ }^{15}$ Varga, A. and Ossmann, D., "LPV-techniques based robust diagnosis of flight actuator faults," Control Engineering Practice, Vol. 31, 2014, pp. 135-147.

${ }^{16}$ Narendra, K. S. and Balakrishnan, J., "Adaptive control using multiple models," IEEE Transactions on Automatic Control, Vol. 42(2), 1997, pp. 171-187.

${ }^{17}$ Ossmann, D., "Optimization based tuning of fault detection and diagnosis systems for safety critical systems," Proc. of IFAC World Congress, Capetown, South Africa, 2014.

${ }^{18}$ Joos, H. D., "Application of Optimization based Worst Case Analysis to Control Law Assessment in Aerospace," Proc. of 3rd EuroGNC, 2015.

19 "BFU Bulletin November 2014," Tech. rep., German Federal Bureau of Aircraft Accident Investigation, 2014.
} 